\title{
SEST observations of southern flat-spectrum radio sources
}

\author{
A.J. Beasley ${ }^{1}$, J.E. Conway ${ }^{2}$, R.S. Booth ${ }^{2}$, L.-Å. Nyman ${ }^{3}$, and M. Holdaway ${ }^{4}$ \\ 1 National Radio Astronomy Observatory, P.O. Box 0, Socorro NM 87801, U.S.A.* \\ 2 Onsala Space Observatory, S-439 92 Onsala, Sweden \\ 3 Swedish-ESO Submillimetre Telescope, European Southern Observatory, Casilla 16001, Santiago 19, Chile \\ 4 National Radio Astronomy Observatory, 949 North Cherry Ave., Campus Building 65, Tucson AZ 85721, U.S.A.
}

Received November 25; accepted December 4, 1996

\begin{abstract}
We present the results of a $2 \& 3 \mathrm{~mm}$ SwedishESO Submillimetre Telescope continuum survey of bright southern flat-spectrum radio sources. Our sample consists of all sources in the PKSCAT90 catalog south of declination $-40^{\circ}$ with accurate positions $\left(<2^{\prime \prime}\right)$, and predicted $3 \mathrm{~mm}$ flux (based on published low-frequency spectral indices) greater than $300 \mathrm{mJy}$ (47 sources). In addition, 32 bright radio sources from the USNO Radio-Optical Reference Frame catalog have been observed. Our overall detection rate for the combined sample is $66 \%$ above typical $5 \sigma$ limits of $300-500 \mathrm{mJy}$. This survey has identified many new sources suitable for use as phase and pointing calibrators for southern millimeter interferometers. A number of individual sources are discussed.
\end{abstract}

Key words: galaxies: active - BL Lacertae objects: general - quasars: general - radio continuum: general; galaxies

\section{Introduction}

The subset of compact extragalactic radio sources which exhibit bright mm-wavelength $(30-300 \mathrm{GHz})$ emission includes many of the most active and interesting objects in the radio sky. These sources usually exhibit flat or inverted spectra $\left(\alpha \geq-0.5, S \propto \nu^{\alpha}\right)$, which are modeled in the context of nuclear jets as a cone of partially optically-thick synchrotron emission with the number density of relativistic particles and magnetic field strength increasing inwards towards an active galactic nucleus (Blandford \& Kon̈igl 1979). High-frequency single-dish monitoring studies (e.g. Brown et al. 1989; Valtaoja et al. 1992a; Tornikoski et al.

Send offprint requests to: A.J. Beasley

* The NRAO is operated by Associated Universities, Inc., under co-operative agreement with the National Science Foundation.
1993; Tornikoski et al. 1996) and ground-based VLBI observations (now routinely available with the Coordinated Millimeter VLBI Array) are providing important information to constrain models of the physical conditions and shock-emission mechanisms in these sources (Marscher \& Gear 1985; Valtaoja et al. 1992b).

Bright compact $\mathrm{mm}$ radio sources can also be used as phase calibrators for interferometer arrays. Planned "fastswitching" schemes involve regular scans of a nearby calibrator source to derive antenna-based phase corrections to reduce or remove the effects of the wet and dry troposphere, and geometric/electronic delays (Holdaway et al. 1995). These schemes require dense grids of bright sources with accurate positions, as residual phase errors increase rapidly with source-calibrator separation and slew time. A survey of 418 northern and equatorial flat and steep-spectrum calibrator candidates was made by Holdaway et al. (1994) using the NRAO $12 \mathrm{~m}$ telescope; in this paper we present the results of a $2 \& 3 \mathrm{~mm}$ continuum survey of 79 southern radio sources, made using the Swedish-ESO Submillimetre Telescope (SEST). The primary goals of this experiment were to catalog bright $\mathrm{mm}$ radio sources in the southern sky suitable for use as pointing and/or phase calibrators, and to identify interesting sources for follow-up radio and optical observations.

\section{The sample}

Our observing sample has two main components. Firstly, we selected the 47 radio sources in the Parkes PKSCAT90 Catalog (Wright \& Otrupcek 1990) which met the following criteria: (1) south of declination $-40^{\circ}$; (2) possessing both 5 \& $8 \mathrm{GHz}$ flux density measurements in PKSCAT90; (3) accurate positions (i.e. quoted errors $<2^{\prime \prime}$ ); and (4) expected $3 \mathrm{~mm}$ flux density greater than $300 \mathrm{mJy}$ (based on a spectral index derived from $5 \& 8 \mathrm{GHz}$ fluxes). The compact (milliarcsecond-scale) structures of most of these sources are not known. Secondly, we selected all sources south of declination $-40^{\circ}$ from the US Naval Observatory (USNO) Radio-Optical Reference Frame (Johnston et al. 
1995). These are compact flat-spectrum radio sources with highly-accurate positions (typically better than 3 milliarcseconds), and typically have $8.4 \mathrm{GHz}$ flux densities greater than $100-200 \mathrm{mJy}$. Of the 60 sources identified this way, 28 were already selected as part of the Parkes sample, leaving 32 new sources. A declination cut-off of $-40^{\circ}$ was used to select regions of the sky inaccessible to northern $\mathrm{mm}$ telescopes. We note that the spectral indices based on non-contemporaneous PKSCAT90 $5 \& 8 \mathrm{GHz}$ flux densities may be significantly in error. We also note that it is possible that a population of radio sources with curved spectra, i.e., which flatten in spectral index or are inverted above $8 \mathrm{GHz}$, could be missed by our criteria.

\section{Observations \& results}

The observations were made during the period 1996 May 21 to May 26 using the $15 \mathrm{~m}$ diameter SEST telescope, situated at the European Southern Observatory Cerro La Silla site (altitude $2300 \mathrm{~m}$ ). A review of the SEST and its capabilities can be found in Booth et al. (1989). We observed using the $2 \mathrm{~mm}$ and $3 \mathrm{~mm}$ SIS receivers simultaneously (single sideband), each coupled to an acousto-optical spectrometer (LR1 and LR2 respectively). A description of these spectrometers can be found in Schieder et al. (1989). The $2 \mathrm{~mm} / \mathrm{LR} 1$ configuration produced 1440 channels (694 kHz separation), centered on $146.969 \mathrm{GHz}$ (the rest frequency of the $\mathrm{CS}(3-2)$ transition). The $3 \mathrm{~mm} / \mathrm{LR} 2$ configuration produced 1600 channels $(681 \mathrm{kHz}$ spacing), centered on $89.188 \mathrm{GHz}$ (rest frequency of $\mathrm{HCO}+(1-0)$ transition). These frequencies were chosen to allow us to search for Galactic CS or HCO+ absorption towards any strongly-detected sources. The receiver temperatures were typically $120-130 \mathrm{~K}$. The primary beam of the SEST telescope has a full-width at half-maximum (FWHM) of $33^{\prime \prime}$ and $55^{\prime \prime}$ at 2 and $3 \mathrm{~mm}$ respectively.

Calibration of the system was performed using the chopper-wheel method (Ulich 1980), which automatically corrects for atmospheric opacity effects. To convert the measured antenna temperatures into flux densities, standard sensitivities of $30 \mathrm{Jy} / \mathrm{K}(2 \mathrm{~mm})$ and $25 \mathrm{Jy} / \mathrm{K}(3 \mathrm{~mm})$ were assumed; these values agreed to within $20 \%$ and $10 \%$ (respectively) with values determined from a single low-elevation observation of Saturn during the run. One 30 minute observation of each source was performed, consisting of 30 cycles of a dual beam-switch mode, where the target is first placed in a signal beam from which a reference beam is subtracted at $6 \mathrm{~Hz}$, then in the reference beam (to remove any residuals due to differences in gain between the beams). The sky separation of the signal and reference beams was $\sim 2.5^{\prime}$. Each half of the cycle produces a spectrum which is the sum of $10 \mathrm{~s}$ of integration on source; these are averaged to produce a flat baseline, and the spectral channels averaged (channels $100-1300$ for LR1, 100 - 1500 LR2). The resultant observing band- widths were $834(2 \mathrm{~mm})$ and $953 \mathrm{MHz}(3 \mathrm{~mm})$. The 30 beamswitch cycles (consisting of approximately $10 \mathrm{~min}$ integration on source) were then averaged; a $1 \sigma$ error was estimated as the standard error of the mean of the individual cycle results. One source $(0454-810)$ was observed on five separate occasions, with consistent results; the value given in Table 1 is the observation with the lowest noise. The zenith opacities at $2 \mathrm{~mm}$ ranged from 0.2 to $<0.02$, with a median value of $\sim 0.05$. The majority of sources were observed at elevations greater than $30^{\circ}$, therefore the small corrections for variations in the telescope gain with elevation at 2 and $3 \mathrm{~mm}$ have not been applied.

In Tables 1 and 2, the results of our observations for the Parkes and USNO samples are presented. In Col. 1, the J2000 source name is given (B1950 names are used in Table 2). Columns 2 and 3 are the source type and redshift (where known) from the NASA/IPAC Extragalactic Database $(\mathrm{Q}=$ quasar, $\mathrm{G}=$ galaxy, $\mathrm{B}=\mathrm{BL}$ Lac $)$, Cols. 4 and 5 are the observed J2000 coordinates, Cols. 6 and 7 are the $2 \mathrm{~mm}$ flux density and error $(1 \sigma)$, and Cols. 8 and 9 are the $3 \mathrm{~mm}$ flux density and error $(1 \sigma)$; all flux densities are given in Jy. Column 10 contains character codes indicating cross-references between the Parkes (P), USNO (U) and (T) Tornikoski et al. (1996) samples. Flux densities preceded by $<$ indicate non-detections at a $5 \sigma$ level. Our overall detection rate for the combined sample is $66 \%$ above typical $5 \sigma$ limits of $300-500 \mathrm{mJy} ; 37$ of 47 sources in the Parkes sample were detected at at least one frequency, and 15 of 32 sources in the USNO sample. We have chosen $5 \sigma$ as a conservative detection limit for these observations, although given the variations in the noise level due to changing atmospheric conditions, it is likely we actually detected sources at lower significance levels, e.g. $4 \sigma$. Inspection of the final summed spectra for each detected source showed no unambiguous examples of Galactic CS or $\mathrm{HCO}+$ absorption.

The expected theoretical thermal noise for our observations was of order $5-10 \mathrm{mJy}$, while in practice the observed $1 \sigma$ noise varied from about $40 \mathrm{mJy}$ to $200 \mathrm{mJy}$, and was roughly correlated with the zenith opacity during the integrations. This discrepancy is most likely due to slight systematic differences in gain between the target and reference beams, combined with temporal and spatial variations in the atmosphere on timescales of the beam switching. The planned replacement of the present chopper wheel system with a rapidly-nutating subreflector with a variable target-reference beam separation may improve the performance of the telescope for continuum surveys.

\section{Individual sources}

Our observations were primarily a finding survey for strong southern millimeter sources, however we now briefly discuss the nature of our detected sources and examine those of special astrophysical interest. 
Table 1. Parkes sample. < indicates non-detection at $5 \sigma$ level. S \& $\sigma$ in Jy

\begin{tabular}{|c|c|c|c|c|c|c|c|c|c|}
\hline Source & Type & $z$ & RA(J2000) & Dec(J2000) & $S_{2 \mathrm{~mm}}$ & $\sigma_{2 \mathrm{~mm}}$ & $S_{3 \mathrm{~mm}}$ & $\sigma_{3 \mathrm{~mm}}$ & ID \\
\hline J0004-4736 & $\mathrm{Q}$ & & $\begin{array}{ll}0 & 435.65\end{array}$ & -473619.6 & $<0.93$ & 0.22 & 1.40 & 0.21 & $\mathrm{P}$ \\
\hline J0051-4226 & Q & 1.74 & 0519.50 & -422633.2 & $<0.18$ & 0.07 & 0.38 & 0.06 & $\mathrm{P}$ \\
\hline J0106-4034 & Q & 0.58 & 1645.10 & -403419.9 & 2.06 & 0.14 & 3.63 & 0.10 & $\mathrm{PU}$ \\
\hline J0210-5101 & Q & 1.00 & 21046.38 & $\begin{array}{lll}-51 & 1 & 1.0\end{array}$ & 2.65 & 0.13 & 3.53 & 0.07 & PTU \\
\hline J0245-4459 & Q & 0.28 & 24554.12 & -445939.7 & 0.49 & 0.09 & 0.45 & 0.05 & $\mathrm{P}$ \\
\hline J0246-4651 & Q & & $246 \quad 0.00$ & -465116.0 & $<0.23$ & 0.13 & 0.62 & 0.06 & $\mathrm{P}$ \\
\hline J0253-5441 & Q & 0.53 & 25329.15 & -544151.3 & 0.63 & 0.12 & 0.94 & 0.07 & $\mathrm{PU}$ \\
\hline J0303-6211 & Q & & $\begin{array}{lll}3 & 350.58\end{array}$ & -621125.0 & 0.60 & 0.09 & 0.83 & 0.10 & $\mathrm{PU}$ \\
\hline J0309-6058 & Q & & $\begin{array}{lll}3 & 9 & 56.03\end{array}$ & -605838.6 & $<0.46$ & 0.13 & 0.88 & 0.08 & $\mathrm{PU}$ \\
\hline J0311-7651 & Q & 0.22 & 31155.50 & -765150.1 & $<0.29$ & 0.13 & $<0.37$ & 0.12 & $\mathrm{PU}$ \\
\hline J0455-4616 & Q & 0.85 & 45551.11 & $-4616 \quad 4.0$ & 0.79 & 0.07 & 1.32 & 0.06 & $\mathrm{PT}$ \\
\hline J0506-6109 & Q & 1.09 & $\begin{array}{ll}5 & 643.90\end{array}$ & $-61 \quad 941.1$ & 0.81 & 0.13 & 1.19 & 0.10 & PTU \\
\hline J0515-4556 & Q & 0.19 & 51545.27 & -455642.8 & $<0.36$ & 0.09 & 0.32 & 0.05 & $\mathrm{P}$ \\
\hline J0522-6107 & Q & 1.40 & 52234.27 & $-61 \quad 758.5$ & $<0.43$ & 0.10 & 0.84 & 0.11 & $\mathrm{PU}$ \\
\hline J0525-4557 & Q & 1.47 & 52531.40 & -455754.7 & $<0.20$ & 0.12 & 0.31 & 0.06 & $\mathrm{P}$ \\
\hline J0538-4405 & Q & 0.89 & 53850.36 & $\begin{array}{lll}-44 & 5 & 8.9\end{array}$ & 3.19 & 0.12 & 4.58 & 0.05 & PTU \\
\hline J0635-7516 & Q & 0.15 & 63546.54 & -751616.8 & 1.01 & 0.10 & 1.76 & 0.16 & PTU \\
\hline J0743-6726 & Q & 1.51 & 74331.52 & -672626.0 & $<-0.13$ & 0.09 & $<0.31$ & 0.08 & PTU \\
\hline J0757-7353 & & & 75714.13 & $-7353 \quad 9.5$ & $<0.03$ & 0.11 & $<-0.01$ & 0.13 & $\mathrm{P}$ \\
\hline J0904-5735 & Q & 0.69 & $9 \quad 453.21$ & $-5735 \quad 3.6$ & $<0.30$ & 0.10 & $<0.20$ & 0.10 & $\mathrm{P}$ \\
\hline J1041-4739 & & & 104144.66 & -473959.6 & $<0.22$ & 0.10 & 0.32 & 0.06 & \\
\hline J1058-8003 & Q & & 105843.69 & $-80 \quad 354.3$ & 0.41 & 0.07 & 1.05 & 0.10 & $\mathrm{PU}$ \\
\hline J1103-5356 & Q & & $11 \quad 352.27$ & -535659.8 & 0.47 & 0.07 & 1.01 & 0.09 & $\mathrm{PU}$ \\
\hline J1107-4449 & Q & 1.59 & $\begin{array}{lll}11 & 7 & 8.69\end{array}$ & $-4449 \quad 7.6$ & 0.79 & 0.10 & 1.58 & 0.07 & $\mathrm{PU}$ \\
\hline J1118-4634 & Q & 0.71 & 111826.92 & -463415.0 & $<0.29$ & 0.09 & $<0.30$ & 0.11 & $\mathrm{PU}$ \\
\hline $\mathrm{J} 1147-6753$ & Q & & 114733.69 & -675341.8 & 1.67 & 0.06 & 2.62 & 0.07 & $\mathrm{P}$ \\
\hline J1224-8313 & & & 122454.49 & -83 1310.4 & $<0.49$ & 0.12 & 0.62 & 0.10 & $\mathrm{P}$ \\
\hline $\mathrm{J} 1255-7138$ & $\mathrm{Q}$ & & 125459.99 & -713820.5 & $<0.15$ & 0.08 & 0.45 & 0.08 & $\mathrm{PU}$ \\
\hline J1424-6807 & Q & & 142455.65 & $-68 \quad 759.2$ & 0.48 & 0.07 & 0.75 & 0.09 & $\mathrm{P}$ \\
\hline $\mathrm{J} 1427-4206$ & Q & 1.52 & 142756.28 & $-42 \quad 618.5$ & 3.34 & 0.09 & 4.83 & 0.12 & PTU \\
\hline J1454-4012 & Q & 1.81 & 145432.89 & -401231.7 & 0.49 & 0.08 & 0.70 & 0.14 & $\mathrm{PU}$ \\
\hline J1624-6809 & $\mathrm{Q}$ & 1.36 & 162418.56 & $\begin{array}{lll}-68 & 9 & 12.8\end{array}$ & $<0.05$ & 0.07 & $<0.08$ & 0.12 & $\mathrm{PU}$ \\
\hline $\mathrm{J} 1723-6500$ & G & 0.01 & 172340.92 & $-65 \quad 035.9$ & $<0.34$ & 0.10 & 0.61 & 0.06 & $\mathrm{PU}$ \\
\hline J1744-5144 & $\mathrm{G}$ & & 174425.41 & -514443.9 & $<0.10$ & 0.20 & $<0.20$ & 0.04 & $\mathrm{P}$ \\
\hline $\mathrm{J} 1803-6507$ & G & & $18 \quad 323.86$ & $-65 \quad 739.4$ & $<-0.13$ & 0.09 & 0.50 & 0.05 & $\mathrm{PU}$ \\
\hline J1809-4552 & & & $18 \quad 957.80$ & -455241.0 & $<0.94$ & 0.20 & 1.67 & 0.18 & $\mathrm{P}$ \\
\hline J1819-5521 & Q & & 181945.44 & -552121.4 & $<0.26$ & 0.05 & 0.32 & 0.06 & $\mathrm{PU}$ \\
\hline $\mathrm{J} 1837-7108$ & Q & 1.35 & 183728.78 & $-71 \quad 841.4$ & 0.73 & 0.07 & 1.17 & 0.10 & $\mathrm{PU}$ \\
\hline J1932-4536 & Q & 0.65 & 193244.90 & -453637.8 & $<0.24$ & 0.07 & 0.42 & 0.04 & $\mathrm{P}$ \\
\hline J1937-3958 & Q & 0.96 & 193716.21 & $-3958 \quad 1.5$ & 0.77 & 0.06 & $<0.39$ & 0.17 & $\mathrm{PT}$ \\
\hline J2009-4849 & Q & 0.07 & $20 \quad 925.40$ & -484953.7 & $<0.41$ & 0.10 & 0.52 & 0.10 & PTU \\
\hline $\mathrm{J} 2207-5346$ & Q & 1.20 & $22 \quad 743.73$ & -534633.8 & $<-0.04$ & 0.21 & $<0.21$ & 0.18 & PTU \\
\hline J2229-4051 & Q & 0.44 & 222918.61 & -405131.7 & $<0.02$ & 0.18 & $<-0.22$ & 0.17 & $\mathrm{P}$ \\
\hline $\mathrm{J} 2235-4835$ & Q & 0.51 & 223513.24 & -483558.5 & 0.73 & 0.14 & 1.20 & 0.10 & $\mathrm{PU}$ \\
\hline J2329-4730 & Q & 1.29 & 232917.71 & -473019.2 & 0.49 & 0.06 & 0.86 & 0.06 & PTU \\
\hline J2336-5236 & Q & & 233611.88 & -523612.9 & $<0.15$ & 0.07 & 0.37 & 0.07 & $\mathrm{P}$ \\
\hline $\mathrm{J} 2357-5311$ & Q & 1.00 & 235753.18 & -531113.8 & $<0.58$ & 0.18 & $<0.48$ & 0.24 & PTU \\
\hline
\end{tabular}

The majority of the detected sources are flat-spectrum core-dominated blazars (either quasars or BL Lac objects) at high redshift (roughly between $z \sim 0.5$ and 2.5). These objects are prime targets for future multi-epoch, multi-frequency monitoring to further constrain the properties of the highly beamed jet emission (Tornikoski et al. 1993). Many of the detected sources are strong at X-ray and gamma-ray wavelengths, and several mechanisms have been proposed for production of this high-energy emission via the inverse-Compton process (Ghisellini \& Madau 1996). These various models predict different relationships between variability in the millimeter and X-ray/gamma-ray, and joint monitoring 
Table 2. USNO sample. < indicates non-detection at $5 \sigma$ level. S \& $\sigma$ in Jy

\begin{tabular}{|c|c|c|c|c|c|c|c|c|c|}
\hline Source & Type & $z$ & $\mathrm{RA}(\mathrm{J} 2000)$ & $\operatorname{Dec}(\mathrm{J} 2000)$ & $S_{2 \mathrm{~mm}}$ & $\sigma_{2 \mathrm{~mm}}$ & $S_{3 \mathrm{~mm}}$ & $\sigma_{3 \mathrm{~mm}}$ & ID \\
\hline$\overline{\mathrm{B} 0047-579}$ & $\mathrm{Q}$ & 1.79 & 04959.47 & -573827.3 & $<0.34$ & 0.10 & 0.60 & 0.06 & $\overline{\mathrm{U}}$ \\
\hline B0056-572 & Q & 0.01 & 05846.58 & -565911.4 & $<0.45$ & 0.15 & 0.56 & 0.08 & $\mathrm{U}$ \\
\hline B0131-522 & Q & 0.01 & $133 \quad 5.76$ & $\begin{array}{lll}-52 & 0 & 3.9\end{array}$ & $<0.28$ & 0.06 & $<0.23$ & 0.08 & $\mathrm{U}$ \\
\hline B0230-790 & Q & 1.07 & 22934.94 & -784745.6 & $<0.03$ & 0.10 & $<0.08$ & 0.09 & $\mathrm{U}$ \\
\hline B0332-403 & Q & 1.44 & 33413.65 & $-40 \quad 825.3$ & 0.73 & 0.12 & 1.21 & 0.07 & UT \\
\hline B0437-454 & & & $439 \quad 0.85$ & -452222.5 & $<0.29$ & 0.07 & $<0.58$ & 0.15 & $\mathrm{U}$ \\
\hline B0438-436 & Q & 2.85 & 44017.17 & $-4333 \quad 8.6$ & $<0.49$ & 0.11 & 1.49 & 0.08 & UT \\
\hline B0454-810 & Q & 0.44 & $450 \quad 5.44$ & $\begin{array}{lll}-81 & 1 & 2.2\end{array}$ & 0.72 & 0.06 & 1.63 & 0.05 & $\mathrm{U}$ \\
\hline B0516-621 & Q & & 51644.92 & $\begin{array}{lll}-62 & 7 & 5.3\end{array}$ & $<0.28$ & 0.29 & $<0.50$ & 0.45 & $\mathrm{U}$ \\
\hline B0530-727 & & & 52930.04 & -724528.5 & $<0.13$ & 0.15 & $<0.24$ & 0.07 & $\mathrm{U}$ \\
\hline B0629-418 & Q & 1.41 & 63111.99 & -415426.9 & $<0.20$ & 0.10 & 0.33 & 0.04 & $\mathrm{U}$ \\
\hline B0738-674 & Q & 1.66 & 73856.49 & -673550.8 & $<0.11$ & 0.08 & $<0.40$ & 0.10 & $\mathrm{U}$ \\
\hline B0823-500 & & & 82526.86 & -501038.4 & $<-0.13$ & 0.08 & $<0.12$ & 0.04 & $\mathrm{U}$ \\
\hline B1105-680 & Q & 0.58 & $11 \quad 712.69$ & -682050.7 & $<0.19$ & 0.08 & 0.53 & 0.06 & $\mathrm{U}$ \\
\hline B1148-671 & Q & & 115113.42 & -672811.0 & $<-0.02$ & 0.06 & $<0.08$ & 0.09 & $\mathrm{U}$ \\
\hline B1236-684 & Q & & 123946.65 & -684530.8 & $<0.16$ & 0.09 & $<-0.05$ & 0.10 & $\mathrm{U}$ \\
\hline B1349-439 & B & 0.05 & 135256.53 & -441240.3 & 0.50 & 0.07 & 0.42 & 0.07 & UT \\
\hline B1549-790 & $\mathrm{G}$ & 0.15 & 155658.86 & $\begin{array}{lll}-79 & 14 & 4.2\end{array}$ & $<0.23$ & 0.09 & 0.63 & 0.08 & UT \\
\hline B1610-771 & Q & 1.71 & 161749.27 & -771718.4 & 0.60 & 0.09 & 1.10 & 0.11 & UT \\
\hline B1903-802 & Q & 0.50 & 191240.01 & $\begin{array}{lll}-80 & 10 & 5.9\end{array}$ & $<0.05$ & 0.12 & 0.51 & 0.09 & $\mathrm{U}$ \\
\hline B1925-610 & Q & & $1930 \quad 6.15$ & $-6056 \quad 9.1$ & $<0.28$ & 0.08 & $<0.23$ & 0.07 & $\mathrm{U}$ \\
\hline B1935-692 & Q & 3.15 & 194025.52 & $-69 \quad 756.9$ & $<0.26$ & 0.08 & $<-0.20$ & 0.07 & $\mathrm{U}$ \\
\hline B1950-613 & Q & & 195510.77 & -611519.1 & $<0.24$ & 0.08 & $<-0.05$ & 0.08 & $\mathrm{U}$ \\
\hline B2052-474 & Q & 1.48 & 205616.35 & -471447.6 & 0.63 & 0.09 & 1.19 & 0.12 & UT \\
\hline B2059-786 & Q & & $21 \quad 544.96$ & -782534.5 & $<0.11$ & 0.10 & $<0.20$ & 0.06 & $\mathrm{U}$ \\
\hline B2106-413 & $\mathrm{Q}$ & 1.05 & $21 \quad 933.18$ & -411020.6 & 1.03 & 0.09 & 1.55 & 0.06 & $\mathrm{U}$ \\
\hline B2109-811 & $\mathrm{G}$ & & 211630.84 & -805355.2 & $<0.07$ & 0.14 & 0.33 & 0.06 & $\mathrm{U}$ \\
\hline B2142-758 & $\mathrm{Q}$ & 1.13 & 214712.73 & -753613.2 & $<0.11$ & 0.10 & $<0.06$ & 0.08 & $\mathrm{U}$ \\
\hline B2146-783 & Q & & $2152 \quad 3.15$ & $\begin{array}{lll}-78 & 7 & 6.6\end{array}$ & $<0.08$ & 0.08 & $<0.08$ & 0.05 & $\mathrm{U}$ \\
\hline B2152-699 & $\mathrm{G}$ & 0.02 & $2157 \quad 5.98$ & -694123.6 & 0.65 & 0.09 & 1.07 & 0.04 & $\mathrm{U}$ \\
\hline B2311-452 & Q & 2.88 & $2314 \quad 9.38$ & -445549.2 & $<-0.18$ & 0.14 & $<0.00$ & 0.07 & $\mathrm{U}$ \\
\hline B2353-686 & Q & 1.71 & $2356 \quad 0.68$ & $-6820 \quad 3.4$ & $<0.64$ & 0.16 & $<0.08$ & 0.14 & $\mathrm{U}$ \\
\hline
\end{tabular}

observations in the different wavebands may distinguish between these mechanisms (e.g. Grandi et al. 1996).

Although inverse-Compton models are generally successful in explaining the spectral energy distributions of blazars, there are sources in which the $\nu F_{\nu}$ spectra peak strongly in the X-ray region; two of these "MeV blazars" are detected in our survey. One is J0210 - 5101 (Blom et al. 1995), and the other is J0506 - 6109 or J0522 - 6107 (Bloeman et al. 1995) (both mm-detected sources are within the COMPTEL error box, although the former is thought more likely as the source of gamma rays). These $\mathrm{MeV}$-peaking sources can be explained by exotic proton-initiated cascade or electron/positron annihilation mechanisms (e.g. Roland \& Hermsen 1995). These authors stress the importance of obtaining VLBI proper motion velocities for these sources which in combination with data on the $\mathrm{MeV}$ peak may strongly constrain the jet physical parameters.

A number of the detected blazars have unusual properties which suggest that it would be worth searching for spectral absorption along the line of sight (e.g., Wiklind \& Combes 1996a). The source B0438 - 436 at $z=2.85$ is one of the highest luminosity sources in the centimeter band (having a flux density of over $7 \mathrm{Jy}$ at $8 \mathrm{GHz}$ ), and shows evidence for strong X-ray absorption (Elvis et al. 1992; Serlemitos et al. 1994), implying a significant foreground column density. Fugmann (1988) noted the extremely high apparent luminosity of this object and the fact that it has many more nearby companion galaxies than would be expected by chance, and argued that gravitational lens amplification might be occurring. It is notable that a number of detected mm-absorption systems have been found toward gravitational lenses (Wiklind \& Combes 1995, 1997). Three blazar objects (J0051 - 4226, J2329 - 4730, and possibly J0635 - 7516) show evidence for optical/uv absorption lines along the line of sight (Junkkarinen et al. 1991), and these might also be searched for molecular absorption, although searches of such systems have proved unsuccessful to date (Wilkind \& Combes 1996a). 
Three of our detected sources are classified as galaxies, with their optical continuum luminosity being dominated by starlight. The $z=0.15$ source B1549 -790 contains a strong compact radio source and has an optical spectrum showing high ionization emission lines and stellar absorption lines characteristic of an evolved stellar population (Tadhunter et al. 1993). The source J1723 - 6500 is identified with the nearby (approximately $80 \mathrm{Mpc}$ ) galaxy NGC 6328, described as high-luminosity elliptical with weak spiral structure (Veron-Cetty et al. 1995); this galaxy is known to be very gas rich, containing over $10^{10}$ solar masses of HI. Australia Telescope images of this gas have been made by Veron-Cetty et al., who speculate that this galaxy is a recent merger product. Given our detection of a strong millimeter continuum source it will be interesting to probe the gas chemistry by looking for millimeter absorption and emission lines. The central continuum source is interesting in its own right, as both the VLBI morphology and the radio spectrum (which peaks in the gigahertz region) are characteristic of so-called Compact Symmetric Objects (Wilkinson et al. 1994), which are thought to be very young radio sources. The detected $3 \mathrm{~mm}$ flux density lies on a smooth extrapolation of the centimeter spectrum and implies a spectral index of -0.7 between $8 \mathrm{GHz}$ and $90 \mathrm{GHz}$. One detection in our sample (J2336 $5236)$ is a known gigahertz-peaked spectrum source (Cersosimo et al. 1994).

The final non-blazar that we have detected is the lobedominated FRII source B2152 -699 at $z=0.028$. This is one of the brightest objects in the southern sky at centimeter wavelengths, and consists of a compact core straddled by much brighter radio lobes (Fosbury et al. 1990; Norris et al. 1990). The pointing position used was that of the compact core as given by astrometric VLBI observations (Johnston et al. 1995), and at $2 \mathrm{~mm}$ in particular most of the extended emission should lie outside of the SEST primary beam. The core has a total flux of $0.82 \mathrm{Jy}$ at $8.4 \mathrm{GHz}$ (Tingay et al. 1996) and hence our 2 and $3 \mathrm{~mm}$ flux densities are consistent with a detection of this flat spectrum core. Optical observations of B2152 - 699 show blue optical emission from a patch of gas oriented almost along the initial radio axis (Tadhunter et al. 1987). It has been argued, based on the high optical polarization of this emission (di Serego Alighieri et al. 1988), that this is evidence for reflected non-isotropic optical emission along the radio axis, and that this object is misdirected quasar (although Tingay et al. investigate the alternative explanation of jet/cloud interactions). Optical spectroscopy of the nucleus (Tadhunter et al. 1993) shows only evidence for a stellar continuum with no nonthermal continuum emission, consistent with blocking of the putative quasar nucleus by a dense torus as proposed by the quasar/radio-galaxy unified scheme. There is, however, some weak broad line emission which may indicate that the source orientation and torus geometry is such that part of the broad-line region can be seen. Given our detec- tion of the nucleus at millimeter wavelengths it will be interesting to perform $\mathrm{cm}$ and $\mathrm{mm}$ spectroscopy to search for absorption from molecular gas in a circumnuclear torus.

\section{Conclusions}

We have described SEST observations to search for mmbright southern radio sources. Combined with source samples in the southern equatorial region (Tornikoski et al. 1996), this sample provides a useful first list of sources for making southern hemisphere pointing observations for single dish telescopes. As noted earlier, the noise level appears to be dominated by spatial and temporal atmospheric fluctuations; the planned replacement of the present SEST chopper wheel system with a nutating subreflector may improve the sensitivity of the telescope for continuum observations.

In the longer term, a large sample of calibration sources with mean separations of only a few degrees will be important for phase-calibration of the proposed southern millimeter interferometers (Woody et al. 1995; Holdaway et al. 1994). At present four southern mm interferometers are under development: the NRAO Millimeter Array $^{1}$, the European Large Southern Array LSA (Booth 1992, 1996), the Japanese LMSA (Ishiguro et al. 1994), and the mm upgrade of the Australia Telescope Compact Array. Site-testing of various locations in the Chilean Atacama Desert is underway for the first three (latitude $-23^{\circ} \mathrm{S}$ ). We note that our survey has identified one pair of bright (approximately $1 \mathrm{Jy}$ at $3 \mathrm{~mm}$ ) sources with separation of only $2^{\circ}$, i.e. J0506-6109/J0522-6107. This pair could be used for making further on-site test observations of phase-calibration methods over angular separations of a few degrees using a single-baseline interferometer with relatively small antennas.

From an astrophysical point of view our sample adds to the list of blazars with detected strong millimeter emission. These sources can be added to existing monitoring programs to improve statistics about flux and spectral variability amongst different classes of beamed sources (Tornikoski et al. 1993). We have identified a number of sources which are circumpolar from southern sites and can be observed continuously in search of intra-day variability (Wagner \& Witzel 1995) at millimeter wavelengths. As described in Sect. 4, a number of the detected sources show evidence for significant foreground column densities either within the host galaxies or elsewhere along the line of sight; these should be searched for centimeter and millimeter absorption lines. Additionally four of our detected sources lie within 10 degrees of the galactic plane (J1041 - 473, J1145 - 676, J1255 - 714 and J1424 - 681) and can be used to search for cold molecular gas within our galaxy (Liszt 1994).

\footnotetext{
$\overline{1}$ http://www.tuc.nrao.edu/mma/mma.html
} 
Acknowledgements. We thank the SEST staff for their assistance during our observing. The SEST is operated jointly by ESO and the Swedish National Facility for Radio Astronomy, Onsala Space Observatory, Chalmers University of Technology. We would like to thank Drs. Mike Kesteven, Miller Goss, and Niven Tasker for their assistance during the early stages of this project. This research has made use of the NASA/IPAC Extragalactic Database which is operated by the Jet Propulsion Laboratory, Caltech with the National Aeronautics and Space Administration.

\section{References}

Blandford R.D., Kon̈igl A., 1979, ApJ 232, 34

Bloeman H., Bennett K., Collmar W., et al., 1995, A\&A 293, L1

Blom J.J., Bennett K., Bloeman H., et al., 1995, A\&A 298, L33

Booth R.S., et al., 1989, A\&A 216, 315

Booth R.S., 1994, in IAU Colloq. 140 "Astronomy with Millimeter and Submillimetre Interferometry", Ishiguro M. and Welch J. (eds.) ASP Conf. Ser. 59, 413

Booth R.S., 1996, in "High Sensitivity Radio Astronomy", Davis R., Jackson N. (eds.). Publ. CUP, 194

Brown L.M.J., et al., 1989, ApJ 340, 129

Cersosimo J.C., Santos M., Cintron S.I., Quiniento Z.M., 1994, ApJS 95, 157

di Serego Alighieri S., Binette L., Courvoisier T.J-L., Fosbury R.A.E., Tadhunter C.N., 1988, Nat 334, 591

Elvis M., Fiore F., Wilkes B., McDowell J., 1994, ApJ 422, 60 Fugmann W., 1988, A\&A 204, 73

Fosbury R.A.E., di Serego Alighieri S., Courvoisier T.J-L., et al., 1990, "Evolution in Astrophysics: IUE Astronomy in the Era of Space Missions", ESA SP 310, 513

Ghisellini G., Madau P., 1996, MNRAS 280, 67

Grandi P., et al., 1996, ApJ 459, 73

Holdaway M.A., Owen F.N., Rupen M.P., 1994, Millimeter Array Memo No. 123, NRAO

Holdaway M.A., Radford S.J.E, Owen F.N., Foster S.M., 1995,
Millimeter Array Memo No. 139, NRAO

Ishiguro M., et al., 1994, in IAU Colloq. 140 "Astronomy with Millimeter and Submillimetre Interferometry", Ishiguro M. and Welch J. (eds.). ASP Conf. Ser. 59

Johnston K.J., et al., 1995, AJ 110, 880

Junkkarinen V., Hewitt A., Burbidge G., 1991, ApJS 77, 203

Liszt H., 1994, ApJ 429, 638

Marscher A.P., Gear W.K., 1985, ApJ 298, 114

Norris R.P., et al., 1990, Proc ASA 8(3), 252

Roland J., Hersen W., 1995, A\&A 297, L9

Schieder R., Tolls V., Winnewisser G., 1989, Exper. Astron. 1, 101

Serlemitos P., Yaoob T., Ricker G., Woo J., Kunieda H., 1994, PASJ 46, L43

Tadhunter C.N., Fosbury R.A.E., Binette L., Danzuger I.J., Robinson A., 1987, Nat 325, 504

Tadhunter C.H., Morganti R., di Serego Alighieri S., Fosbury R.A.E., Danziger I.J., 1993, MNRAS 263, 999

Tingay S.J., et al., 1996, AJ 111, 718

Tornikoski M., et al., 1993, AJ 105, 1680

Tornikoski M., et al., 1996, A\&AS 116, 157

Ulich B.L., 1980, Astrophys. Lett. 21, 21

Valtaoja E., et al., 1992a, A\&A 254, 80

Valtaoja E., et al., 1992b, A\&A 254, 71

Veron-Cetty M.-P., Woltjer L., Ekers R.D., Stavely-Smith L., 1995, A\&A 297, L79

Wagner S.J., Witzel A., 1995, ARA\&A 33, 163

Wiklind T.T., Combes F., 1995, A\&A 299, 382

Wiklind T.T., Combes F., 1996a, Nat 379, 139

Wiklind T.T., Combes F., 1997, A\&A (in press)

Wilkinson P.N., Polatadis A., Readhead A.C.S., Xu W., Pearson T.J., 1994, ApJ 432, L87

Woody D., Holdaway M., Lay O., Masson C., Owen F., Plambeck D., Radford S., Sutton E., 1995, Millimeter Array Memo No. 144, NRAO

Wright A.E., Otrupcek R., 1990, PKSCAT90 Parkes Radio Sources Catalogue, Version 1.01, Australia Telescope National Facility 